\title{
AURKA induces EMT by regulating histone modification through Wnt/ $\beta$-catenin and PI3K/Akt signaling pathway in gastric cancer
}

\author{
Xi Liu ${ }^{1}$, Zhaoxia Li ${ }^{1}$, Yue Song ${ }^{1}$, Rui Wang ${ }^{1}$, Lei Han ${ }^{3}$, Qixue Wang ${ }^{2,3}$, Kui Jiang ${ }^{1}$, \\ Chunsheng Kang ${ }^{2,3}$, Qingyu Zhang ${ }^{1}$ \\ ${ }^{1}$ Department of Gastroenterology, Tianjin Medical University General Hospital, Tianjin 300052, China \\ ${ }^{2}$ Department of Neurosurgery, Tianjin Medical University General Hospital, Heping District, Tianjin 300052, China \\ ${ }^{3}$ Laboratory of Neuro-Oncology, Tianjin Neurological Institute, Tianjin 300052, China \\ Correspondence to: Qingyu Zhang, e-mail: zhangqy@tijmu.edu.cn \\ Chunsheng Kang, e-mail: kang97061@gmail.com \\ Keywords: AURKA, Wnt/ß-catenin pathway, PI3K/AKt pathway, histone modification \\ Received: November 11,2015 Accepted: March 28, $2016 \quad$ Published: April 21, 2016
}

\section{ABSTRACT}

Gastric cancer, a highly invasive and aggressive malignancy, is the third leading cause of death from cancer worldwide. Genetic association studies have successfully revealed several important genes consistently associated with gastric cancer to date. However, these robust gastric cancer-associated genes do not fully elucidate the mechanisms underlying the development and progression of the disease. In the present study, we performed an alternative approach, a gene expressionbased genome-wide association study (eGWAS) across 13 independent microarray experiments (including 251 gastric cancer cases and 428 controls), to identify top candidates $(p<0.00001)$. Additionally, we conducted gene ontology analysis, pathway analysis and network analysis and identified aurora kinase A (AURKA) as our candidate. We observed that MLN8237, which is a specific inhibitor of AURKA, decreased the $\beta$-catenin and the phosphorylation of Akt1 and GSK-3 $\beta$, as well as blocked the Akt and Wnt signaling pathways. Furthermore, MLN8237 arrested the cells in the G2/M phase. The activity of Wnt and Akt signaling pathways affected the level of histone methylation significantly, and we supposed that MLN8237 affected the level of histone methylation through these two signaling pathways. Additionally, the treatment of MLN8237 influenced the level of H3K4 me1/2/3 and H3K27 me1/2/3. Chip data on cell lines suggested that MLN8237 increases the level of H3K27 me3 on the promoter of Twist and inhibits EMT (epithelial-mesenchymal transition). In summary, AURKA is a potential therapeutic target in gastric cancer and induces EMT through histone methylation.

\section{INTRODUCTION}

Gastric cancer is a highly invasive and aggressive malignancy and one of the leading causes of death from cancer worldwide; therefore, investigations into its initiation and development are of great importance $[1-3]$. The EMT is important during carcinogenesis and metastatic progression, and this term has been more liberally referred as a recognizable change in cellular phenotype characterized by the loss of cell junctions and gain of migratory behaviors. Recent studies have established that aberrant EMT activation in the human stomach is closely associated with gastric carcinogenesis and tumor progression $[4,5]$. The prognosis of gastric cancer patients usually depends on the early detection and treatment of malignant tumor characteristics such as invasion and metastasis, which are the primary causes of treatment failure. Therefore, the exploration of gastric cancer initiation and progression mechanisms may improve early diagnosis and treatment efficacy. 
In the past years, numerous genome-wide association studies (GWASs) have revealed significant associations of single-nucleotide polymorphisms SNPs with susceptibility to gastric cancer and have refreshed the genetic knowledge of gastric cancer [6-10]. Recently, there were several studies aimed to discover candidate (SNPs) for gastric cancer, such as SNPs at 1q22, 10q23 and 5p13.1 [11-14]. However, there have been few fully identified, functionally important genes in the pathogenesis of gastric cancer because many genes are often detected as significant in each microarray experiment, and it is difficult to subselect optimal candidates from individual studies for further verification.

Our hypothesis is that those genes most repeatedly implicated across a large set of experimental representations of gastric cancer can serve as data-driven causal gastric cancer genes and candidates for validation. The method is viable because many of these sources are publicly available; we selected 13 independent microarray experiments for gastric cancer. The identification was followed by confirmatory functional studies using assays in vitro and mouse models.

A wealth of epigenomic data has identified abnormal regulation of epigenetic processes as a prominent theme. Recurrent somatic alterations involved in DNA methylation, post-translational histone modification and chromatin remodeling have highlighted the importance of the epigenetic regulation of gene expression in the initiation and maintenance of various malignancies [15-18]. However, the mechanisms of malignant transformation driven by aberrant epigenetic regulators require a thorough understanding. In the present study, we identified the most significant candidate gene from gastric cancer and normal gastric mucosa and researched the mechanisms underlying the initiation of gastric cancer, and identified the aberrant epigenetic regulations. This study offers the opportunity to gain insight into key genes, key pathways and nodes of epigenetic regulation, further enhancing our ability to deliver effective novel compounds for clinical target therapy.

\section{RESULTS}

\section{eGWASs identify AURKA as a functional candidate gene for gastric cancer}

We performed eGWASs for gastric cancer using 13 independent microarray experiments, and 679 samples were collected from public repositories. Additionally, we ranked all 30,663 genes by the likelihood that repeated differential expression for that gene was due to chance, and then controlled for Fisher's exact test. To overview which molecular functions were most shared in the highest ranked genes in our gastric cancer eGWAS, we took 184 genes (Bonferroni threshold, $P<1.0 \times 10^{-5}$ ) from our
eGWAS (Figure 1A, Table S1). Next, we estimated the enrichment of Gene Ontology (GO) terms. Interestingly, "cell cycle" and "collagen" functions were the most implicated of the top-ranked genes (Figure 1B). We then performed KEGG pathway analysis and calculated the enrichment $P$-value (Table S2). However, there was no implicated enrichment in any signaling pathway. Finally, we conducted network analysis, which integrated the experiments, database and literature, and the results demonstrated that AURKA is the center of the network (Figure 1C, 1D).

Our gastric cancer candidate gene, AURKA (Figure 1; Fisher's exact test; $\left.\mathrm{P}=6.83 \times 10^{-6}\right)$, was markedly differentially expressed in experiments studying gastric cancer with normal gastric mucosa. AURKA is located on chromosome 20q13 and encodes a centrosomeassociated, cell cycle-regulated serine/threonine kinase involved in mitosis [22]. Previous studies have revealed that the overexpression of AURKA results in centrosome amplification and cytokinesis failure, causing aneuploidy [23]. Furthermore, AURKA regulates several important proteins such as AKT, $\beta$-catenin and p53 in cancer cells [24-28]. The findings suggest that AURKA might stimulate the generation of gastric cancer.

\section{MLN8237 decreased the phosphorylation of p-Akt1 p-GSK-3 $\beta$ and $\beta$-catenin and suppressed cell viability and invasive growth}

To validate the effect of MLN8237 on gastric cancer and glioblastoma cell lines, Western blotting results demonstrated the reduced expression of $\beta$-catenin and phosphorylation of $\mathrm{p}-\mathrm{AKT} 1$ and $\mathrm{p}-\mathrm{GSK}-3 \beta$. However, the expression of AURKA was slightly increased. These results confirmed that MLN8237 suppressed the activity of the Wnt $/ \beta$-catenin and PI3K/Akt signaling pathways (Figure 2A). Furthermore, to determine whether the treatment of MLN8237 was accompanied by a change of EMT, we detected the expression of E-cadherin, N-cadherin and Twist following MLN8237 treatment (Figure 2A). The expression of E-cadherin was increased, the expression of $\mathrm{N}$-cadherin and Twist was decreased in the MLN8237-treated cells.

The cell viability of MGC-803, BGC-823, SGC7901 and U87 EGFRvIII cell lines was measured by calculating the cell cycle distribution following MLN8237 treatment (Figure 2B). As shown in Figure 2B, the G0/G1 phase fractions of the control MGC-803, BGC-823, SGC7901 and U87 EGFRvIII cells were 54.42\%, 77.47\%, $61.99 \%$ and $53.25 \%$, respectively. However, treatment with MLN8237decreased the percentage of cells in the G0/G1 phase to $51.09 \%, 45.21 \%, 0.82 \%$ and $13.73 \%$, respectively. Additionally, the $\mathrm{G} 2 / \mathrm{M}$ phase fractions in the control cells were $8.97 \%, 5.11 \%, 10.37 \%$ and $9.12 \%$, respectively. However, MLN8237 treatment increased the 
percentage of cells in the $\mathrm{G} 2 / \mathrm{M}$ phase to $17.85 \%, 18.45 \%$, $87.65 \%$ and $80.67 \%$, respectively. The results suggested that MLN8237 treatment arrested the cells in the G2/M phase. As shown in Figure 3C, treatment with MLN8237 significantly suppressed the invasion of cells compared with that of the control group $(\mathrm{P}<0.01)$.

\section{The Wnt/ $\beta$-catenin signaling pathway regulates the methylation and acetylation of $\mathrm{H} 3 \mathrm{~K} 4$ and H3K27}

Above study have demonstrated a significant decrease in $\beta$-catenin and the phosphorylation of GSK$3 \beta$ following MLN8237 treatment. AURKA was shown to increased $\beta$-catenin and phosphorylation of GSK-3 $\beta$, as well as the activity of the $\mathrm{Wnt} / \beta$-catenin signaling pathway. To investigate the role of the Wnt/ $\beta$-catenin signaling pathway in regulating histone methylation, we performed Western blot to analyze the change in histone methylation following treatment with FH535 and Wnt3a (Figure 3).

FH535 is a small-molecule inhibitor of the $\beta$-catenin/TCF4 complex. In MGC-803 and BGC-823 gastric cancer cells, the levels of $\mathrm{H} 3 \mathrm{~K} 4 \mathrm{me} 1 / \mathrm{me} 2$ and H3K27 me3 were increased, those of H3K4 me3 and
H3K27 me1/2 were decreased at $20 \mu \mathrm{M}$ FH535 and continued to increase at $40 \mu \mathrm{M}$ FH535 in a dose-dependent manner after FH535 treatment for 24 hours. Furthermore, the changes were consistent with the prolongation of the time of FH535 treatment. Consistent with the findings above, the methylation of $\mathrm{H} 3 \mathrm{~K} 4$ and $\mathrm{H} 3 \mathrm{~K} 27$ tended to be uniform in Hep-3B hepatocarcinoma cells, colon cancer Caco 2 cells and glioma U87and U87 EGFRvIII cells.

Wnt3a, a canonical Wnt ligand, activates the canonical $\mathrm{Wnt} / \beta$-catenin signaling pathway. To evaluate whether the change in the methylation of $\mathrm{H} 3 \mathrm{~K} 4$ and $\mathrm{H} 3 \mathrm{~K} 27$ was relevant to the status of the $\mathrm{Wnt} / \beta$-catenin signaling pathway, we enhanced $\mathrm{Wnt} / \beta$-catenin signaling using exogenous Wnt3a. In MGC-803 and BGC-823 gastric cancer cells, Hep-3B hepatocarcinoma cells and Caco 2 colon cancer cells, the levels of $\mathrm{H} 3 \mathrm{~K} 4 \mathrm{me} 1 / 2$ and H3K27 me3 were decreased, and those of H3K4 me3 and H3K27 me1/2 were increased with $100 \mathrm{ng} / \mathrm{ml} \mathrm{Wnt3a}$ treatment for 24 hours.

TGF- $\beta 1$ is one of the most well-known inducers of EMT and binds to its receptors on the cell membrane to phosphorylate Smads. We cultured MGC-803 and BGC823 cells with FH535 at 20 and $40 \mu \mathrm{M}$ following treatment with TGF- $\beta 1$ for 24 hours. The alterations were evident

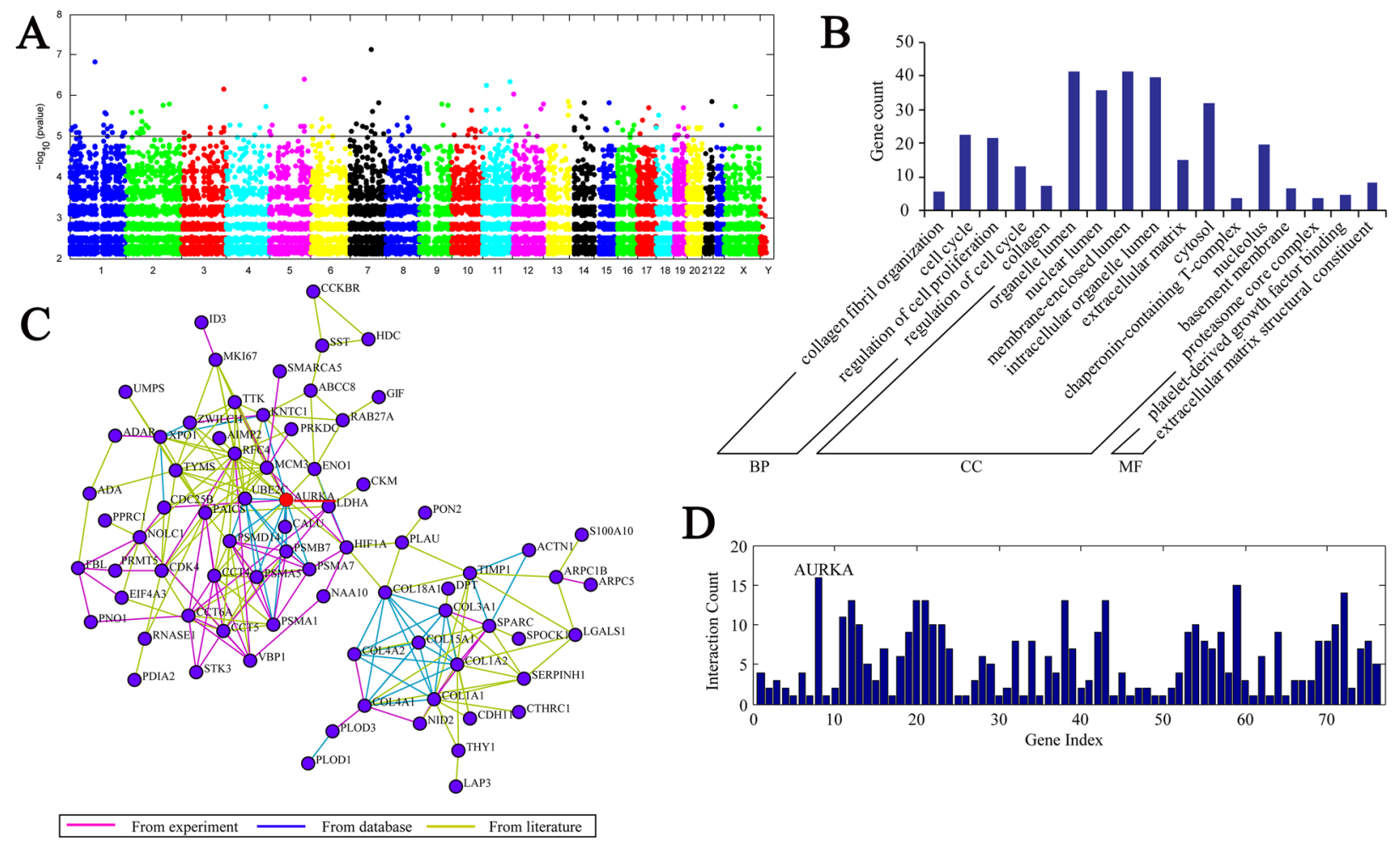

Figure 1: A. eGWAS for gastric cancer using a Fisher's exact test. Plot of $-\log 10(P$ value $)(y$ axis $)$ by chromosomal position $(x$ axis $) . P$ values for each gene were calculated from our eGWAS across 13 microarray experiments with 679 gastric cancer case-control microarray samples(251 cases and 428 controls). The red line indicates $P=0.00001$, and there were 184 genes $P<0.00001$. B. These 184 differential genes were categorized into three groups - biological process, cellular component and molecular function — through gene ontology analysis. The results showed that there was enrichment in the cell cycle and collagen. C. D. Network analysis demonstrated that AURKA is the center of the network and consisted of 184 differential genes. 
after EMT was induced by TGF- $\beta 1$ and were consistent with the results with FH535 treatment only.

\section{The PI3K/Akt signaling pathway regulates the methylation and acetylation of $\mathrm{H} 3 \mathrm{~K} 4$ and $\mathrm{H} 3 \mathrm{~K} 27$}

The above findings demonstrated a significant decrease in p-Akt1 following MLN8237 treatment. AURKA regulates p-Akt1 and the activity of thePI3K/Akt signaling pathway. To investigate the role of the PI3K/ Akt signaling pathway in regulating histone modification, we performed Western blotting to analyze the change in histone methylation following treatment with LY294002 and EGF (Figure 4).

Previous studies have shown that LY294002, which suppresses PI3K, can inhibit the expression of p-Akt in cancer. In MGC-803 and BGC-823 gastric cancer cells,
Hep-3B hepatocarcinoma cells and Caco 2 colon cancer cells, the levels of H3K4 me1/2/3 and H3K27 me2/3 were increased, and those of $\mathrm{H} 3 \mathrm{~K} 27$ me1 were decreased following treatment with $10 \mu \mathrm{M}$ LY294002. There was no change noted LY294002 after $20 \mu \mathrm{M}$ treatment. However, in U87 glioma cells, the levels of H3K4 me1/2 and H3K27 me 2 were increased, and those of $\mathrm{H} 3 \mathrm{~K} 4 \mathrm{me} 3$ and $\mathrm{H} 3 \mathrm{~K} 27$ me1/3 were decreased following treatment with 10 and $20 \mu \mathrm{M}$ LY294002.

EGF is an upstream activator of the AKT pathway. To evaluate whether the change in the methylation and acetylation of $\mathrm{H} 3 \mathrm{~K} 4$ and $\mathrm{H} 3 \mathrm{~K} 27$ was relevant to the status of the PI3K/Akt signaling pathway, we enhanced PI3K/Akt signaling with exogenous EGF. The levels of H3K4 me1/2/3 and H3K27 me2/3 were decreased, and those of H3K27 mel were increased in MGC-803, BGC-823, Hep-3B and Caco 2 cells following treatment with $100 \mathrm{ng} / \mathrm{ml} \mathrm{EGF}$.
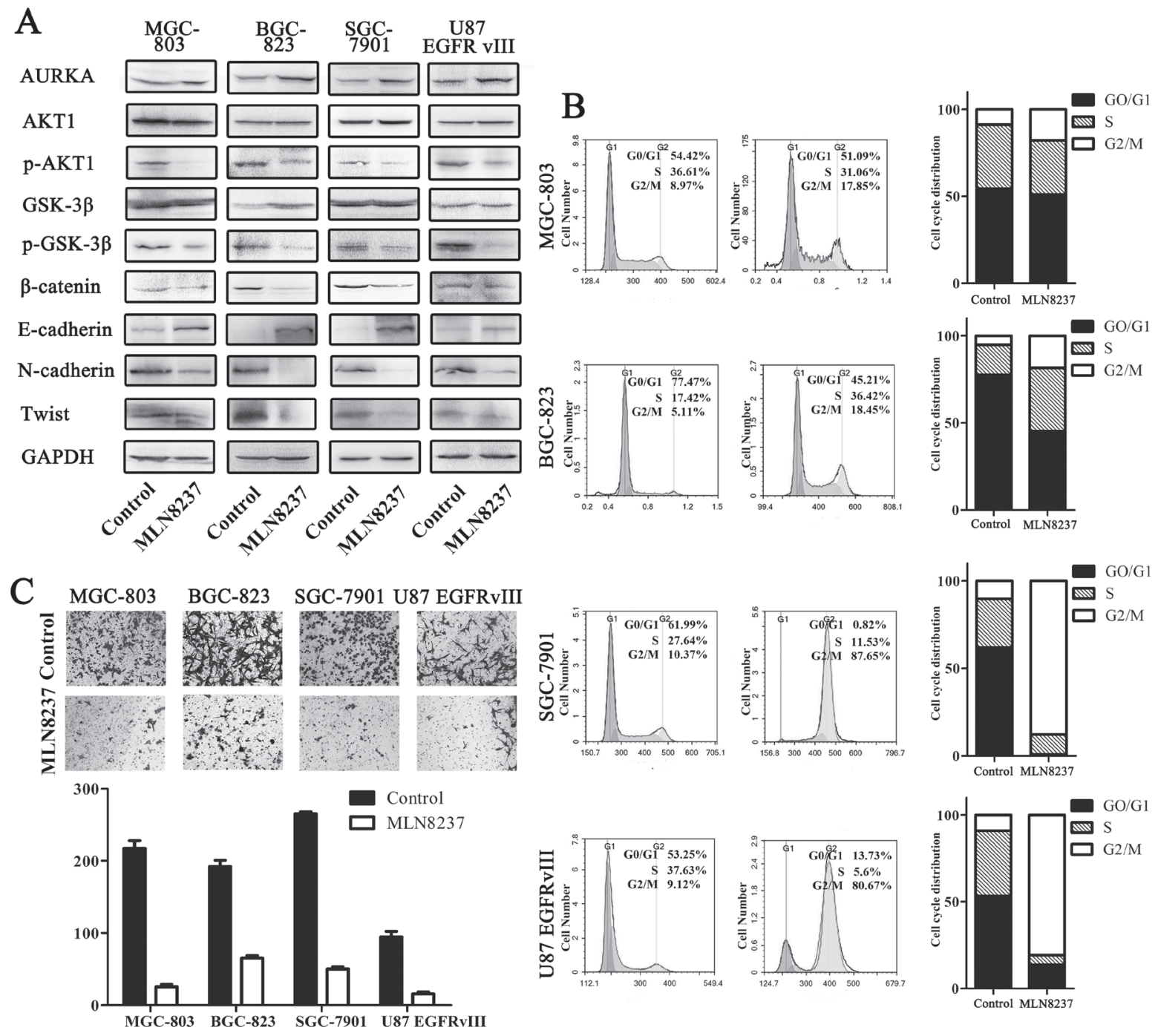

Figure 2: MLN8237 blocked the Wnt and Akt signaling pathways and induced the arrest of cells in the G2/M phase. A. $\beta$-catenin and related target protein expression were assessed by Western blot analysis. GAPDH was, respectively, used as the total protein loading control. B. The cell cycle distributions were detected by flow cytometry. The data revealed a significantly increased percentage of cells in the G2/M phase in all four cell lines. C. Transwell assays for assessing the invasion of the MLN8237-treated cells $(\mathrm{P}<0.01)$. 


\section{MLN8237 regulates the modification of histone and increases the expression of $\mathrm{H3K27}$ me3 in the Twist promoter}

The results demonstrated an increased expression of H3K27 me2/3 in the gastric cancer cell lines MGC803, BGC-823 and SGC-7901. However, the expression of H3K4 me1/2/3 and H3K27 me1 showed no consistent tendency following MLN8237 treatment in these gastric cancer cell lines. Furthermore, the results showed decreased expression of $\mathrm{H} 3 \mathrm{~K} 4 \mathrm{me} 1 / 3$ and $\mathrm{H} 3 \mathrm{~K} 27 \mathrm{me} 1 / 3$ in U87 EGFRvIII cells. The effect on histone methylation in gastric cancer cells was very different in U87 EGFRvIII cells(Figure 5A).

To examine the functional role of $\mathrm{H} 3 \mathrm{~K} 27$ me3 in the promoter region of Twist, we performed ChIP with quantitative PCR analysis (ChIP-qPCR). The results showed that the level of $\mathrm{H} 3 \mathrm{~K} 27 \mathrm{me} 3$ in the promoter of Twist increased following MLN8237 treatment $(\mathrm{P}<0.01$,
Figure 5B). H3K27 me3 is a repressive histone marker, and the results above suggest that the level of Twist was decreased.

\section{Inhibition of AURKA suppresses gastric cancer and glioblastoma growth in xenograft models}

MLN8237 treatment suppresses gastric cancer and glioma tumorigenesis in vitro. To verify the role of AURKA, we established a subcutaneous gastric carcinoma model using MGC-803 cells as described previously. Seven days after implantation, DMSO and MLN8237 were intraperitoneally injected every two days for 21 days. The mouse and tumor weights were measured, and the tumor volume was measured using calipers to measure the tumor length and width (Figure 6A). Treatment with MLN8237 suppressed the growth of gastric cancer cells compared with the DMSO-treated group significantly. Additionally, to confirm the role of AURKA, we established a subcutaneous
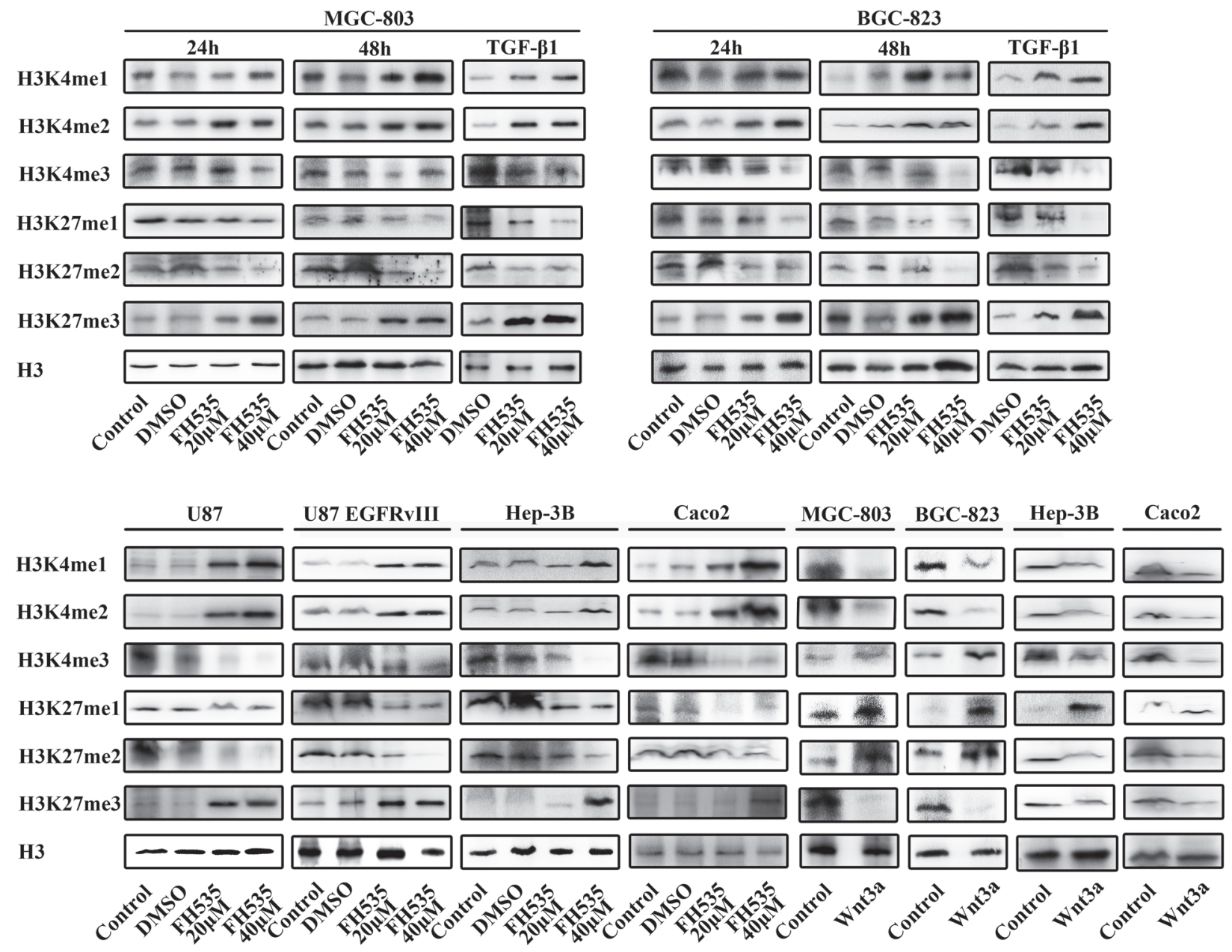

Figure 3: The Wnt// -catenin signaling pathway regulates histone methylation. The expression levels of $\mathrm{H} 3 \mathrm{~K} 4 \mathrm{me} 1 / \mathrm{me} /$ me3 and H3K27 me1/me2/me3 were assessed by Western blot analysis. Histone H3 was used as the nuclear extraction loading control.

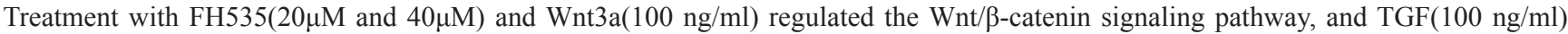
induced the process of EMT. 
gastric carcinoma model using the MGC-803 cell line and an orthotopic glioma model using the U87 EGFRvIII cell line. Compared with the Lenti-NC-treated MGC-803 cells, the Lenti-siAURKA-treated tumor was suppressed significantly (Figure 6B). Bioluminescence imaging showed tumor stasis in the Lenti-siAURKA group on day 14, and the results demonstrated prolonged survival by KaplanMeier survival curves $(P<0.05$; Figure $6 C)$. In addition, immunohistochemistry was used to compare the H3K27 me3 and Ki67 expression levels in these groups. For cell proliferation markers, Lenti-siAURKA treatment decreased the expression of Ki67. MLN8237 and Lenti-siAURKA treatment increased the expression of $\mathrm{H} 3 \mathrm{~K} 27$ me 3 in gastric carcinoma models (Figure 6D). However, Lenti-siAURKA treatment decreased the expression of H3K27 me3 in the glioblastoma intracranial model, and the change worked together with the result shown in Western blot analysis.

\section{H3K27 me3 expression accumulates in gastric cancer}

Immunostaining analysis revealed $\mathrm{H} 3 \mathrm{~K} 27$ me3 protein expression in gastric cancer, and the expression levels were significantly greater in gastric adenocarcinoma than in normal and paracarcinoma tissues (Table 1, Figure S1). However, age and gender were not significantly associated with clinical outcome.

Previous studies indicated that MLN8237 inhibited the Wnt and Akt signaling pathways. Thus, we measured whether MLN8237 whether MLN8237 affect $\beta$-catenin
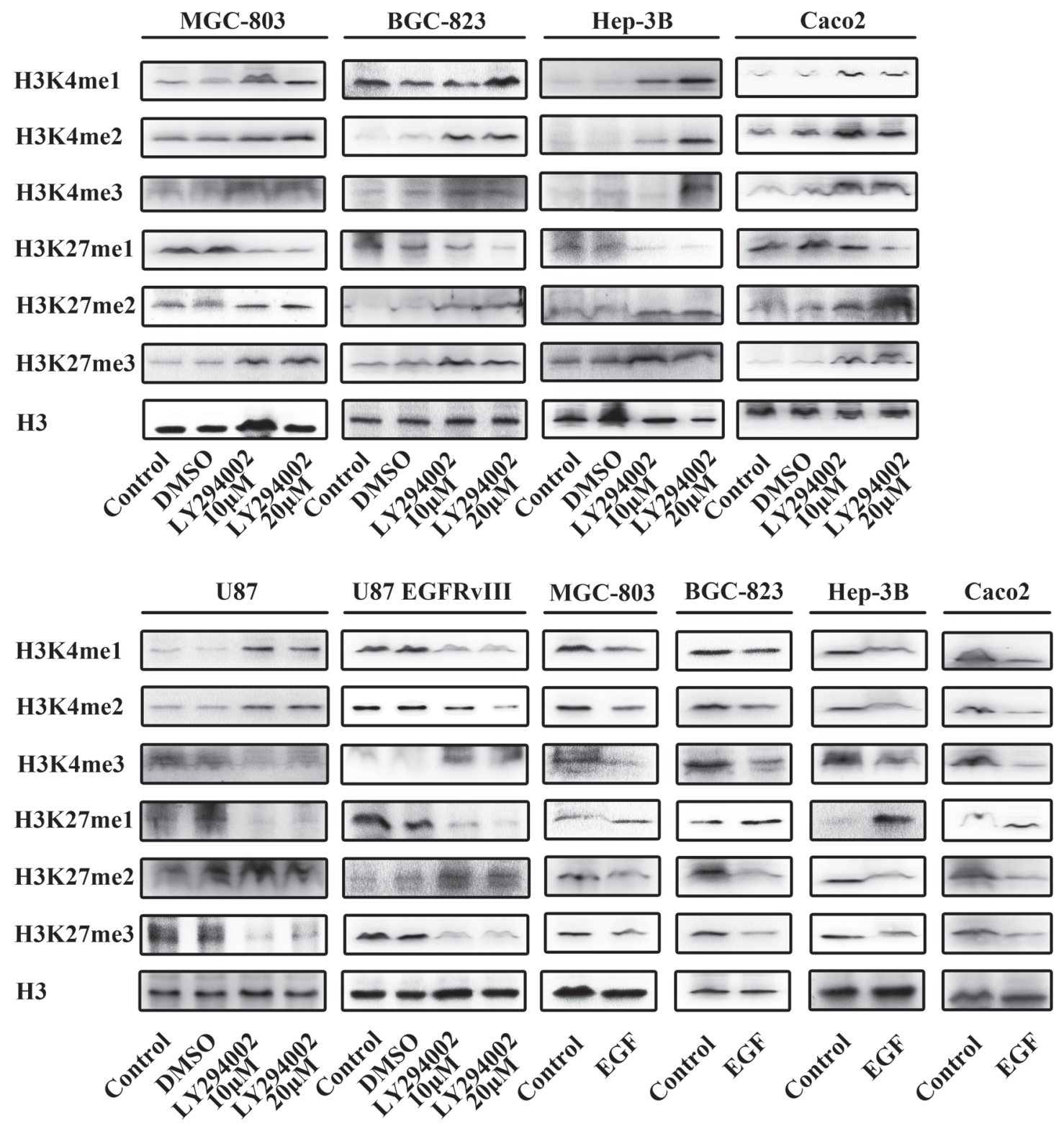

Figure 4: The Akt/PI3K signaling pathway promotes histone methylation. Treatment with $L Y 294002(10 \mu \mathrm{M}$ and $20 \mu \mathrm{M})$ and EGF(100 ng/ml) affected the Akt/PI3K signaling pathway. 
transcriptional activity by the TOP/FOR flash reporter plasimid. Decreased activity of the $\beta$-catenin/TCF4 in MLN8237-treatde cells was detected in MGG-803, BGC-823, SGC-7901 and U87 EGFRvIII cells $(\mathrm{P}<0.05$, Figure S2).

\section{DISCUSSION}

The generation of gastric cancer is a multifactorial and complicated process. Although much has been learned concerning the genetic and biochemical bases of gastric cancer because of the difficulties to identify and validate therapeutic targets, few novel therapeutic targets have been identified [29-31]. Recently, several groups have published GWASs of gastric cancer focusing on singlenucleotide polymorphisms (SNPs), such as in a locus on chromosome 10q23 in the PLCE1 gene or in PRKAA1 at 5p13.1 [23, 24, 32]. However, few functionally important genes have been identified in the pathogenesis of gastric cancer.

In our gastric cancer eGWAS, we identified 184 genes as significantly repeatedly dysregulated with $\mathrm{P}<1.0 \times 10^{-}$ ${ }^{5}$ (under the Bonferroni-corrected threshold). Using GO enrichment analysis, we found that these genes were implicated in the "cell cycle" and "collagen". However, there was no enrichment in pathway analysis. Furthermore, our network analysis demonstrated that AURKA is the pivotal gene in the study. The role of AURKA during mitosis has been defined to a large extent [33]. Furthermore, the involvement of AURKA in the cell cycle and cell division, as well as its role in other cell signaling pathways, is well established. In addition to its well-defined role in mitosis, the overexpression of AURKA and upregulation of its enzymatic activity have been linked to tumorigenesis,
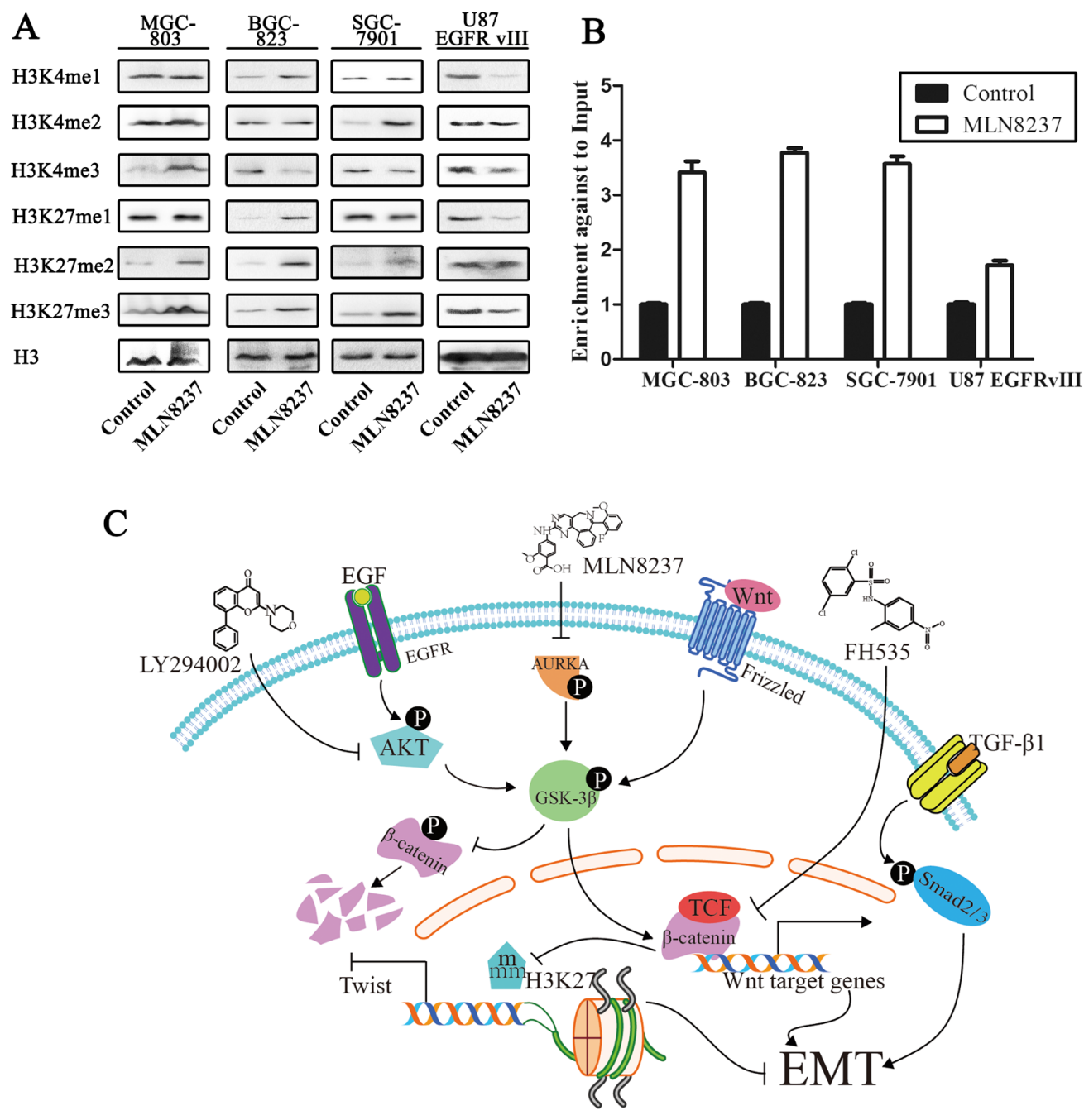

Figure 5: The change in histone methylation of $\mathrm{H3K} 4$ and $\mathrm{H} 3 \mathrm{~K} 27$ is assessed by Western blot analysis. A. H3 was, respectively, used as the nuclear extraction protein loading control. B. ChIP demonstrated that the level of H3K27 me3 in the Twist promoter was increased following MLN8237 treatment $(\mathrm{P}<0.01)$. C. MLN8237 suppresses the Wnt/ $\beta$-catenin and PI3K/Akt signaling pathways through inhibition of the phosphorylation of GSK-3 $\beta$, and inhibition of the EMT process through increasing the level of H3K27 me3 in the Twist promoter. 
specifically in ovarian, prostate, esophageal, gastric, colon and breast cancers. Additionally, a specifically inhibitor of AURKA has been designed [34-39].

Results from previous studies have suggested that the overexpression of AURKA increases the level of p-GSK$3 \beta$, p-Akt1 and $\beta$-catenin and plays an important role in cell proliferation, tumor progression and metastasis [40-42]. MLN8237 is a specific inhibitor of AURKA [43]. And our study have showed that MLN8237 decreased the level of p-GSK-3 $\beta$, p-Akt1 and $\beta$-catenin in our study. Additionally, MLN8237 treatment arrested the cells in the G2/M phase and suppressed the invasive ability of cells [44-47].
Chromatin is a complex of DNA and histone proteins, and the term "epigenetics" is defined to describe heritable changes in a cellular phenotype that are independent of alterations in the DNA sequence [48]. The information conveyed by epigenetic modifications plays a critical role in the regulation of all DNA-based processes, such as transcription, DNA repair and replication, and histone modification is an important part of epigenetic modification [49]. Additionally, many histone modifications are misregulated in cancer. The $\mathrm{Wnt} / \beta$-catenin and PI3K/Akt signaling pathways play important roles in the development of cancer, and it is important to determine the relationship
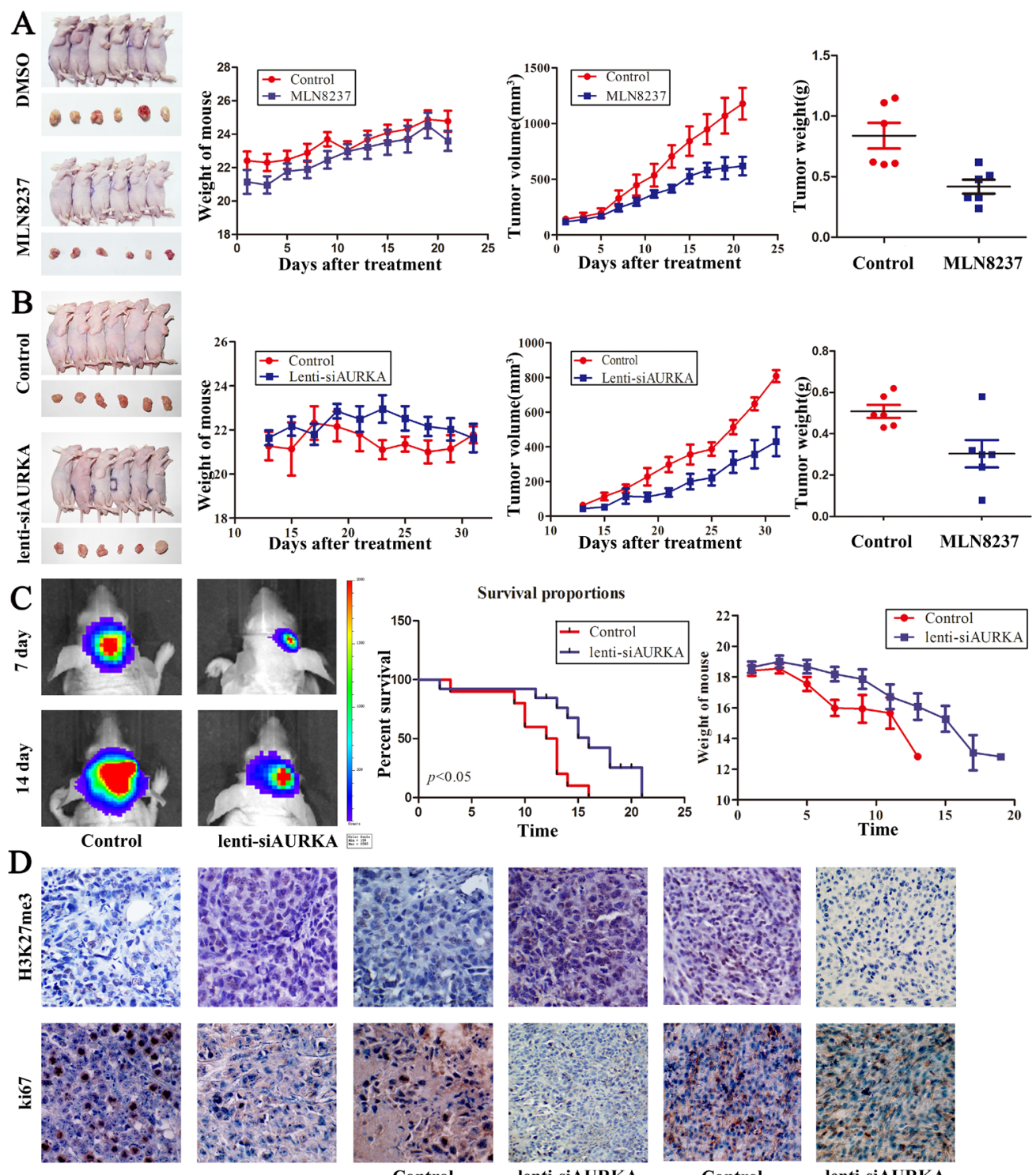

MLN8237

Control

lenti-siAURKA

Control

lenti-siAURKA

Figure 6: Treatment with MLN8237 and the decreased level of AURKA suppress gastric cancer and glioblastoma growth in vivo. A, B. Mice and tumor samples from the MGC-803/DMSO, MGC-803/MLN8237, MGC-803/Control and MGC-803/ lenti-siAURKA groups. The mouse body weights, and tumor growth and weight were evaluated using the in vivo proliferation assay. C. Bioluminescent images from the control and lenti-siAURKA animals at 14 and 21 days after tumor implantation. Contrasting survival benefits were observed in mice, and mouse body weights were evaluated. D. Representative photomicrographs of immunohistochemistry for H3K27 me3 and ki67 on implanted tumor sections. 
Table 1: Immunostaining analysis revealed the expression levels of H3K27me3 in gastric adenocarcinoma, paracarcinoma and normal tissues

\begin{tabular}{|c|c|c|c|c|c|c|c|c|}
\hline Variable & - & + & ++ & +++ & Positive rate & Cases & $\chi^{2}$ value & $P$ value \\
\hline \multicolumn{9}{|l|}{ Gender } \\
\hline Male & 12 & 36 & 14 & 4 & $81.8 \%$ & 66 & \multirow{2}{*}{1.844} & \multirow{2}{*}{0.605} \\
\hline Female & 9 & 8 & 6 & 3 & $65.4 \%$ & 26 & & \\
\hline \multicolumn{9}{|l|}{ Age, year } \\
\hline$\leq 60$ & 6 & 31 & 11 & 7 & $89.1 \%$ & 55 & \multirow{2}{*}{3.271} & \multirow{2}{*}{0.352} \\
\hline$>60$ & 10 & 19 & 6 & 2 & $73 \%$ & 37 & & \\
\hline \multicolumn{9}{|l|}{ Pathology } \\
\hline Normal & 0 & 3 & 7 & 0 & 1 & 10 & \multirow{3}{*}{15.598} & \multirow{3}{*}{$0.004 *$} \\
\hline Paracarcinoma & 0 & 8 & 21 & 8 & 1 & 37 & & \\
\hline Adenocarcinoma & 0 & 4 & 17 & 24 & 1 & 45 & & \\
\hline
\end{tabular}

between the activities of the Wnt and Akt signaling pathways and histone modification [50]. Anti-epigenetic therapy that targets the Wnt/ $\beta$-catenin and EGFR pathways is vital for therapy [51]. We proposed the application of FH535, LY294002, Wnt3a and EGF, and we found the relationship between the activities of the Wnt and Akt pathways and histone methylation. KDM6A and KDM6B are important demethylases that affect signaling pathways important for endoderm differentiation, such as the Wnt and Akt signaling pathways. However, the mechanism concerning the interplay between histone methylation and the two signaling pathways remains unclear.

AURKA is a crucial candidate gene for gastric cancer and is relevant to the $\mathrm{Wnt} / \beta$-catenin and PI3K/Akt signaling pathways. We suspected that AURKA regulates modification through the effects on the $\mathrm{Wnt} / \beta$-catenin and PI3K/Akt signaling pathways. The results above showed that MLN8237 increased the expression of H3K27 me2/ me3/AC in the gastric cancer cell lines MGC-803 and BGC-823, and SGC-7901 decreased the expression of H3K4 me1/me3 and H3K27 mel/me3 in U87 EGFRvIII cells. According to previous studies, H3K4 is associated with active genes in euchromatin, whereas H3K27 is associated with heterochromatic regions of the genome. Histone methylation plays an important role in diverse biological processes, including transcription, and can function in both gene activation and repression [14-16]. Additionally, different methylation states on the same residue can also localize differently. H3K4 me2/3 usually spans the transcriptional start site of active genes, whereas $\mathrm{H} 3 \mathrm{~K} 4 \mathrm{mel}$ is a modification associated with active enhancers, and trimethylation of $\mathrm{H} 3 \mathrm{~K} 27$ is associated with gene repression [52].

Twist is a crucial gene in the development of the embryo and stimulate the process of EMT [53]. Epigenetic silencing has been studied as a potential candidate for targeted therapy. ChIP analysis showed that the level of
H3K27 me3 in the promoter of Twist increased following MLN8237 treatment, and we suspect that epigenetic regulation was an approach of MLN8237 function. And MLN8237 decreased Twist and inhibited EMT. A number of prior studies have suggested that EMT is associated with increased resistance of cancer cells to anticancer drugs [54]. But statistically significant positive correlations between expression levels of the epithelial biomarker E-cadherin and drug sensitivity was found for only ten out of 118 drugs [55]. We need more efforts to research whether inhibition of AURKA could damage gastric cancer's drug resistance by reverse EMT. The blocking of AURKA suppressed the activity of the two signaling pathways and inhibited the expression of EMT-related genes, including Twist, through regulating histone modification. As we all know, AURKA is not only correlated with the Wnt and Akt signaling pathways but also may regulate histone modification through other ways. Additionally, the mechanism underlying the effect on histone modification by AURKA was not investigated, and we need to identify more downstream genes related to AURKA to clarify the effect of AURKA on gastric cancer. In conclusion, the study provides insights into the relevance of AURKA and related genes for the progression of EMT, reveals the significance of histone modification in the progression and highlights their potential to be exploited as therapeutic targets.

\section{MATERIALS AND METHODS}

\section{eGWAS}

All of the gastric cancer-related, genome-wide microarray experiments used in this study were collected from the NCBI Gene Expression Omnibus (GEO, www. ncbi.nlm.mih.gov/geo) public database. There were 679 samples (428 gastric cancer cases and 251 controls) in 13 independent databases. To estimate differences between 
the groups of samples from diabetic subjects and groups representing the control, raw post quantitation microarray data were reanalyzed using Significance Analysis of Microarrays software (SAM). There are 30,663 genes in the database. For each gene, we counted the observed number of microarray experiments in which the gene was significantly dysregulated. We next calculated $P$ values from the number of positive/negative experiments for each gene and sums of the number of positive/negative experiments for all of the other genes using Fisher's exact test as an alternative. The Bonferroni threshold $\left(P=1.0 \times 10^{-5}\right)$ indicated that there were 184 gastric cancer susceptibility genes.

We applied these 184 genes to the gene ontology database, and divided them into the following three parts: biological process, cellular component, molecular function through GSEABase. Next, we performed enrichment analysis across DAVID online tools. Furthermore, we applied these genes to the KEGG pathway database through GneMAPP v2.1 and calculated the enrichment $P$-value. Finally, we performed network analysis across medusa, including the KEGG database, experiments and the literature.

\section{Cell culture}

The human gastric cancer cell linesMGC-803, BGC-823, and SGC-7901, hepatocarcinomacell line Hep$3 \mathrm{~B}$, colon cancer cell line ( $\mathrm{Caco} 2)$ and glioblastoma cell lineU87 were obtained from the China Academia Sinica Cell Repository, Shanghai, China. The human glioblastoma cell line U87 EGFRvIII was donated by the Laboratory of Neurooncology, Tianjin Neurological Institute (Tianjin, China). U87vlll cells carry a truncated mutant EGFR gene, which can be activated without EGF stimulation consistently [19]. Cells were cultured in DMED/1640 medium supplemented with $10 \% \mathrm{FBS}$ and incubated at $37^{\circ} \mathrm{C}$ in $5 \% \mathrm{CO}_{2}$.

\section{Chemical reagents and antibodies}

For in vitro studies, stock solutions of MLN8237 (20 mmol/L), FH535 (10 mmol/L) and LY294002 (20 $\mathrm{mmol} / \mathrm{L}$ ) were prepared in DMSO. Stock solutions of recombinant human Wnt3a $(100 \mu \mathrm{g} / \mathrm{ml})$, EGF $(100 \mu \mathrm{g} /$ $\mathrm{ml})$ and TGF- $\beta 1(100 \mu \mathrm{g} / \mathrm{ml})$ were diluted in phosphatebuffered saline (PBS).

Dimethyl sulfoxide (DMSO), LY294002 and EGF were purchased from Sigma. FH535 was purchased from Merck. Wnt3a was purchased from abnova. MLN8237 was purchased from selleck.

AURKA, $\beta$-catenin, AKT1, p-AKT1, GSK-3 $\beta$, p- GSK-3 $\beta$, Twist, H3K4 me3/AC and H3K27 me2/me3/ AC antibodies were purchased from Abcam. E-cadherin, N-cadherin, H3K4 me1/me2 and H3K27 mel antibodies were purchased from Cell Signaling Technology. The Ki67 antibody was purchased from Santa Cruz Biotechnology. The H3 antibody was purchased from Ray Antibody. The GAPDH antibody was purchased from Zhongshan Bio Corp.

\section{Western blot analysis}

The cells were harvested at $24 \mathrm{~h}$ after the treatment of MLN8237, and the total protein was extracted using RIPA lysis buffer containing proteinase inhibitors. The homogenates were clarified by centrifugation at $12,000 \times \mathrm{g}$ for $15 \mathrm{~min}$ at $4^{\circ} \mathrm{C}$. Nuclear protein was extracted using the Nuclear and Cytoplasmic Protein Extraction Kit (Beyotime Biotechnology), according to the manufacturer's protocols. Next, the protein concentration was measured using the bicinchoninic acid method. A total of $40 \mu \mathrm{g}$ of protein mixed with $4 \times$ SDS loading buffer was loaded into each lane and separated by $10 \%$ or $15 \%$ sodium dodecyl sulfatepolyacrylamide gel electrophoresis (SDS-PAGE). The separated proteins were transferred to PVDF membranes (Millipore), and the membranes were incubated in the above described primary antibodies, followed by incubation with HRP-conjugated secondary antibody (Zhongshan Bio Corp, Beijing, China). Protein expression was visualized using the SuperSignal protein detection kit (Pierce, USA). The membrane was stripped and reprobed with Histone H3 (Ruikang, 1:2000 dilution), GAPDH primary antibody (Zhongshan Bio Corp, Beijing, China) as a control.

\section{Cell cycle analysis}

The effect of MLN8237 on cell cycle distribution was determined by flow cytometry. Briefly, MGC-803, BGC-823, SGC-7901 and U87 EGFRvIII were treated with MLN8237 at $10 \mu \mathrm{M}$ for 24 hours. Cells in the growth $\log$ phase were harvested, washed with PBS, fixed with $70 \%$ ethanol overnight at $4^{\circ} \mathrm{C}$, and then incubated with RNase at $37^{\circ} \mathrm{C}$ for $30 \mathrm{~min}$. The cell nuclei were stained with propidium iodide for an additional $30 \mathrm{~min}$. Cells were examined in a flowcytometer, and the results are presented as the percentage of cells in each phase.

\section{Transwell invasion assay}

The ability of cells to migrate was assessed using matrigel-coated transwell membranes (Becton-Dickinson). After 30 min of incubation at $37^{\circ} \mathrm{C}$, the matrigel solidified and served as an extracellular matrix for tumor cell invasion analysis. The cells were then incubated for 48 hours at $37^{\circ} \mathrm{C}$ in $5 \% \mathrm{CO}_{2}$. Cell invasion to the underside of the matrigel-coated membrane was detected by staining cells with crystal violet, counting them and then imaging them under an inverted microscope at $\times 200$ magnification (Olympus Corp, Tokyo, Japan). The results were expressed as the average number of invasive cells per field.

\section{Chromatin immunoprecipitation (ChIP)}

To test whether there was $\mathrm{H} 3 \mathrm{~K} 27$ me3 bound to the promoter of Twist, cells were treated with DMSO or MLN8237 for 24 hours prior to formaldehyde fixation. Next, cells were harvested for chromatin 
immunoprecipitation (ChIP) using the EZ-ChIP kit (Millipore), according to the manufacturer's protocols. Chromatin was extracted, and cross-linked DNA was cut to approximately 200-1000 base pairs. Protein G Agarose was added to the antibody/chromatin complexes and incubated overnight at $4^{\circ} \mathrm{C}$. $\mathrm{H} 3 \mathrm{~K} 27$ me 3 antibodies were used to pull down DNA from formaldehyde cross-linked chromatin. The protein G Agarose-antibody/chromatin complex was resuspended in wash buffer and centrifuged to collect the protein/DNA complex. The protein/DNA cross-links were reversed to obtain free DNA. Purified DNA was quantified using real-time quantitative PCR.

\section{Animal experiments}

BALB/c-A nude mice at 4 weeks of age for animal experiments were purchased from the Animal Center of the Cancer Institute, Chinese Academy of Medical Science. We estimated the therapeutic potential of MLN8237 using xenograft models and MGC-803 gastric cancer cells. Additionally, the mice were randomly assigned to four groups (six subcutaneous tumors/group). In Group $1,100 \mu \mathrm{L}$ of DMSO/DMEM (1:1) was intraperitoneally injected into the xenograft tumor model. In Group 2, MLN8237 (20 mg/kg/dose) dissolved in $100 \mathrm{ml} \mathrm{DMSO/}$ DMEM was intraperitoneally injected every 2 days during the experimental period.

To test the effect of knocking down AURKA, lentiviruses containing an AURKA inhibitor sequence (Lenti-siAURKA) or negative control (Lenti-NC) were obtained from Shanghai Genechem, China. Additionally, $2 \times 10^{6}$ MGC-803 cells and $10^{5}$ U87 EGFRvIII cells stably expressing Lenti-siAURKA were injected into the peritoneal cavity, and the brain underwent intraperitoneal and intracranial inoculation. The animal groups were as follows: group 3: MGC-803 cells expressing Lenti-NC; group 4: MGC-803 cells expressing Lenti-siAURKA; group 5: U87 EGFRvIII cells expressing Lenti-NC; group 6: U87 EGFRvIII cells expressing Lenti-siAURKA. The tumor volumes were quantified using calipers to measure the tumor length and width. The weight of the mice was measured every 2 days, and the tumor weight was surveyed at the endpoint of the study. Mice in groups 5 and 6 were imaged for Fluc activity using bioluminescence imaging as described previously [20]. The weight of mice was measured every 2 days.

\section{Immunohistochemistry staining}

Tissue microarrays were purchased from Alenabio, and the specimens were classified according to World Health Organization (WHO) categories (2007) under an institutional review board. Samples included 6cases of WHO Grade I gastric adenocarcinoma, 30 cases of WHO Grade II gastric adenocarcinoma, 9 cases of WHO Grade III gastric adenocarcinoma and 55 cases of normal gastric mucosa.
For immunohistochemical staining, formalin-fixed tissue samples were prepared as paraffin-embedded sections, and immunostaining was performed on sections using the avidin-biotin-complex method. Primary antibodies specific for H3K27 me3 (Abcam; 1:100 dilution) and Ki67 (Santa Cruz; 1:100 dilution) were diluted in PBS with $0.1 \%$ Tween 20 and incubated overnight at $4^{\circ} \mathrm{C}$. The sections were incubated in biotinylated secondary antibodies (Zhongshan Biology; $1: 100$ dilution) at $37^{\circ} \mathrm{C}$ for 1 hour, followed by incubation in avidin-biotin complex solution for an additional 1 hour. Protein expression was detected by coloration with diaminobenzidine (DAB) buffer, and the sections were counterstained with hematoxylin. Staining results were scored by two pathologists. Sections with no labeling, or with $<5 \%$ labeled cells, were score as 0 . Sections with $5-30 \%$ of positive cells were scored as 1 , sections with $31-70 \%$ of positive cells were scored as 2 , and sections with $\geq 71 \%$ of positive cells were scored as 3 . The staining intensity was scored similarly, as described previously [21].

\section{Statistical analysis}

The significance of Kaplan-Meier statistics was tested using the log-rank test. Multivariate analysis was performed using the multivariate Cox regression model. SPSS 16.0 (SPSS, Chicago, IL) was used for all of the calculations. All of the data were represented by the mean \pm SD. Statistical significance was determined at $P<0.05$.

\section{ACKNOWLEDGMENTS}

This research work supported by funds from China National Natural Scientific Found (81172356) and National High Technology Research and Development Program 863 (2014AA021102).

\section{CONFLICTS OF INTEREST}

The authors declare no conflicts of interest.

\section{REFERENCES}

1. Herrero, R., J.Y. Park, D. Forman, The fight against gastric cancer - the IARC Working Group report. Best Pract Res Clin Gastroenterol. 2014;28:1107-14.

2. Hunt RH, Camilleri M, Crowe SE, El-Omar EM, Fox JG, Kuipers EJ, Malfertheiner P, McColl KE, Pritchard DM, Rugge M, Sonnenberg A, Sugano K, Tack J. The stomach in health and disease. Gut 2015;64:1650-68.

3. Yeh JM, Hur C, Ward Z, Schrag D, Goldie SJ. Gastric adenocarcinoma screening and prevention in the era of new biomarker and endoscopic technologies: a costeffectiveness analysis. Gut. 2016;65:563-74.

4. Tsuji, T., S. Ibaragi, G.F. Hu, Epithelial-mesenchymal transition and cell cooperativity in metastasis. Cancer Res, 2009. 69: 7135-9. 
5. Roussos, E.T., et al., AACR special conference on epithelial-mesenchymal transition and cancer progression and treatment. Cancer Res, 2010. 70: 7360-4

6. Hu N, Wang Z, Song X, Wei L, Kim BS, Freedman ND, Baek J, Burdette L, Chang J, Chung C, Dawsey SM, Ding $\mathrm{T}$, Gao YT, et al. Genome-wide association study of gastric adenocarcinoma in Asia: a comparison of associations between cardia and non-cardia tumours. Gut. 2015; pii: gutjnl-2015-309340.

7. Oh S, Oh S. Epidemiological and genome-wide association study of gastritis or gastric ulcer in korean populations. Genomics Inform 2014;12:127-33.

8. Kang M, Ding X, Xu M, Zhu H, Liu S, Wang M, Wu D, Tong N, Gong W, Zhou J, Zhang Z. Genetic variation rs10484761 on 6 p21.1 derived from a genome-wide association study is associated with gastric cancer survival in a Chinese population. Gene 2014;536:59-64.

9. Saeki N, Ono H, Sakamoto H, Yoshida T. Genetic factors related to gastric cancer susceptibility identified using a genome-wide association study. Cancer Sci 2013;104:1-8.

10. Shi Y, Hu Z, Wu C, Dai J, Li H, Dong J, Wang M, Miao X, Zhou Y, Lu F, Zhang H, Hu L, Jiang Y, et al. A genomewide association study identifies new susceptibility loci for non-cardia gastric cancer at 3q13.31 and 5p13.1. Nat Genet 2011;43:1215-8.

11. Sakamoto H, Yoshimura K, Saeki N, Katai H, Shimoda T, Matsuno Y, Saito D, Sugimura H, Tanioka F, Kato S, Matsukura N, Matsuda N, Nakamura T, et al. Genetic variation in PSCA is associated with susceptibility to diffuse-type gastric cancer. Nat Genet 2008;40:730-40.

12. Zheng L, Zhu C, Gu J, Xi P, Du J, Jin G. Functional polymorphism rs4072037 in MUC1 gene contributes to the susceptibility to gastric cancer: evidence from pooled 6,580 cases and 10,324 controls. Mol Biol Rep 2013;40:5791-6.

13. Labrador L, Torres K, Camargo M, Santiago L, Valderrama E, Chiurillo MA. Association of common variants on chromosome 8q24 with gastric cancer in Venezuelan patients. Gene 2015;566:120-4.

14. Mocellin S, Verdi D, Pooley KA, Nitti D. Genetic variation and gastric cancer risk: a field synopsis and meta-analysis. Gut 2015;64:1209-19.

15. Bird A. DNA methylation patterns and epigenetic memory. Genes Dev 2002;16:6-21.

16. Greer EL, Shi Y. Histone methylation: a dynamic mark in health, disease and inheritance. Nat Rev Genet 2012;13:343-57.

17. Watson IR, Takahashi K, Futreal PA, Chin L. Emerging patterns of somatic mutations in cancer. Nat Rev Genet 2013;14:703-18.

18. Dawson MA, Kouzarides T. Cancer epigenetics: from mechanism to therapy. Cell 2012;150:12-27.

19. Zheng Q, Han L, Dong Y, Tian J, Huang W, Liu Z, Jia X, Jiang T, Zhang J, Li X, Kang C, Ren H. JAK2/STAT3 targeted therapy suppresses tumor invasion via disruption of the EGFRvIII/JAK2/STAT3 axis and associated focal adhesion in EGFRvIII-expressing glioblastoma. Neuro Oncol.2014; 16:1229-43.

20. Shi Z, Zhang J, Qian X, Han L, Zhang K, Chen L, Liu J, Ren Y, Yang M, Zhang A, Pu P, Kang C. AC1MMYR2, an inhibitor of dicer-mediated biogenesis of Oncomir miR-21, reverses epithelial-mesenchymal transition and suppresses tumor growth and progression. Cancer Res 2013;73:5519-31.

21. Shi Z, Qian X, Li L, Zhang J, Zhu S, Zhu J, Chen L, Zhang K, Han L, Yu S, Pu P, Jiang T, Kang C. Nuclear translocation of beta-catenin is essential for glioma cell survival. J Neuroimmune Pharmacol 2012;7:892-903.

22. Giet R, Prigent C. Aurora/Ipllp-related kinases, a new oncogenic family of mitotic serine-threonine kinases. J Cell Sci 1999;112:3591-601.

23. Zhou H, Kuang J, Zhong L, Kuo WL, Gray JW, Sahin A, Brinkley BR, Sen S. Tumour amplified kinase STK15/ BTAK induces centrosome amplification, aneuploidy and transformation. Nat Genet 1998;20:189-93.

24. Dar AA, Zaika A, Piazuelo MB, Correa P, Koyama T, Belkhiri A, Washington K, Castells A, Pera M, El-Rifai W. Frequent overexpression of Aurora Kinase A in upper gastrointestinal adenocarcinomas correlates with potent antiapoptotic functions. Cancer 2008;112:1688-98.

25. Yang H, He L, Kruk P, Nicosia SV, Cheng JQ. Aurora-A induces cell survival and chemoresistance by activation of Akt through a p53-dependent manner in ovarian cancer cells. Int J Cancer 2006;119:2304-12.

26. Damalas A, Ben-Ze'Ev A, Simcha I, Shtutman M, Leal JF, Zhurinsky J, Geiger B, Oren M. Excess beta-catenin promotes accumulation of transcriptionally active p53. Embo J 1999;18:3054-63.

27. Morin PJ, Sparks AB, Korinek V, Barker N, Clevers H, Vogelstein B, Kinzler KW. Activation of beta-catenin-Tcf signaling in colon cancer by mutations in beta-catenin or APC. Science 1997;275:1787-90.

28. Polakis P. The oncogenic activation of beta-catenin. Curr Opin Genet Dev 1999;9:15-21.

29. Wu WK, Cho CH, Lee CW, Fan D, Wu K, Yu J, Sung JJ. Dysregulation of cellular signaling in gastric cancer. Cancer Lett 2010;295:144-53.

30. Saka M, Morita S, Fukagawa T, Katai H. Present and future status of gastric cancer surgery. Jpn J Clin Oncol 2011;41:307-13.

31. Nobili S, Bruno L, Landini I, Napoli C, Bechi P, Tonelli F, Rubio CA, Mini E, Nesi G. Genomic and genetic alterations influence the progression of gastric cancer. World J Gastroenterol 2011;17:290-9.

32. Song HR, Kim HN, Kweon SS, Choi JS, Shim HJ, Cho SH, Chung IJ, Park YK, Kim SH, Choi YD, Joo KW, Shin MH. Common genetic variants at 1q22 and 10q23 and gastric cancer susceptibility in a Korean population. Tumour Biol 2014;35:3133-7.

33. Mahankali M, Henkels KM, Speranza F, GomezCambronero J. A non-mitotic role for Aurora kinase A as a 
direct activator of cell migration upon interaction with PLD, FAK and Src. J Cell Sci 2015;128:516-26.

34. Do TV, Xiao F, Bickel LE, Klein-Szanto AJ, Pathak HB, Hua X, Howe C, O'Brien SW, Maglaty M, Ecsedy JA, Litwin S, Golemis EA, Schilder RJ, et al. Aurora kinase A mediates epithelial ovarian cancer cell migration and adhesion. Oncotarget. 2016. doi: 10.18632/oncotarget. 7335 .

35. Gritsko TM, Coppola D, Paciga JE, Yang L, Sun M, Shelley SA, Fiorica JV, Nicosia SV, Cheng JQ. Activation and overexpression of centrosome kinase BTAK/Aurora-A in human ovarian cancer. Clin Cancer Res 2003;9:1420-6.

36. Li JJ, Weroha SJ, Lingle WL, Papa D, Salisbury JL, Li SA. Estrogen mediates Aurora-A overexpression, centrosome amplification, chromosomal instability, and breast cancer in female ACI rats. Proc Natl Acad Sci U S A 2004;101:18123-8.

37. Tong T, Zhong Y, Kong J, Dong L, Song Y, Fu M, Liu Z, Wang M, Guo L, Lu S, Wu M, Zhan Q. Overexpression of Aurora-A contributes to malignant development of human esophageal squamous cell carcinoma. Clin Cancer Res 2004;10:7304-10.

38. Buschhorn HM, Klein RR, Chambers SM, Hardy MC, Green S, Bearss D, Nagle RB. Aurora-A over-expression in high-grade PIN lesions and prostate cancer. Prostate 2005;64:341-6.

39. Baba Y, Nosho K, Shima K, Irahara N, Kure S, Toyoda S, Kirkner GJ, Goel A, Fuchs CS, Ogino S. Aurora-A expression is independently associated with chromosomal instability in colorectal cancer. Neoplasia 2009;11:418-25.

40. Dar AA, Belkhiri A, El-Rifai W. The aurora kinase A regulates GSK-3beta in gastric cancer cells. Oncogene 2009;28:866-75.

41. Xu LZ, Long ZJ, Peng F, Liu Y, Xu J, Wang C, Jiang L, Guo T, Kamran M, Li SS, Wang CL, Wang HJ, Zhao YF, et al. Aurora kinase a suppresses metabolic stress-induced autophagic cell death by activating mTOR signaling in breast cancer cells. Oncotarget 2014;5:7498-511. doi: 10.18632/oncotarget.2241.

42. Jia L, Lee HS, Wu CF, Kundu J, Park SG, Kim RN, Wang LH, Erkin OC, Choi JS, Chae SW, Yang HB, Choi YL, Shin YK. SMAD4 suppresses AURKA-induced metastatic phenotypes via degradation of AURKA in a TGFbetaindependent manner. Mol Cancer Res 2014;12:1779-95.

43. Nair JS, Schwartz GK. MLN-8237; A dual inhibitor of aurora A and B in soft tissue sarcomas. Oncotarget 2016.

44. Thrane S, Pedersen AM, Thomsen MB, Kirkegaard T, Rasmussen BB, Duun-Henriksen AK, Laenkholm AV, Bak M, Lykkesfeldt AE, Yde CW. A kinase inhibitor screen identifies Mcl-1 and Aurora kinase A as novel treatment targets in antiestrogen-resistant breast cancer cells. Oncogene 2015;34:4199-210.

45. Melichar B, Adenis A, Lockhart AC, Bennouna J, Dees EC, Kayaleh O, Obermannova R, DeMichele A, Zatloukal P, Zhang B, Ullmann CD, Schusterbauer C. Safety and activity of alisertib, an investigational aurora kinase A inhibitor, in patients with breast cancer, small-cell lung cancer, non-small-cell lung cancer, head and neck squamous-cell carcinoma, and gastro-oesophageal adenocarcinoma: a fivearm phase 2 study. Lancet Oncol 2015;16:395-405.

46. Li JP, Yang YX, Liu QL, Pan ST, He ZX, Zhang X, Yang T, Chen XW, Wang D, Qiu JX, Zhou SF. The investigational Aurora kinase A inhibitor alisertib (MLN8237) induces cell cycle G2/M arrest, apoptosis, and autophagy via p38 MAPK and Akt/mTOR signaling pathways in human breast cancer cells. Drug Des Devel Ther 2015;9:1627-52.

47. Sehdev V, Katsha A, Ecsedy J, Zaika A, Belkhiri A, El-Rifai W. The combination of alisertib, an investigational Aurora kinase A inhibitor, and docetaxel promotes cell death and reduces tumor growth in preclinical cell models of upper gastrointestinal adenocarcinomas. Cancer 2013;119:904-14.

48. Hanzelmann S, Beier F, Gusmao EG, Koch CM, Hummel S, Charapitsa I, Joussen S, Benes V, Brummendorf TH, Reid G, Costa IG, Wagner W. Replicative senescence is associated with nuclear reorganization and with DNA methylation at specific transcription factor binding sites. Clin Epigenetics 2015;7:19.

49. Wang Z, Dao R, Bao L, Dong Y, Wang H, Han P, Yue $\mathrm{Y}, \mathrm{Yu} \mathrm{H}$. Epigenetic reprogramming of human lung cancer cells with the extract of bovine parthenogenetic oocytes. J Cell Mol Med 2014;18:1807-15.

50. Davidson KC, Adams AM, Goodson JM, McDonald CE, Potter JC, Berndt JD, Biechele TL, Taylor RJ, Moon RT. Wnt/beta-catenin signaling promotes differentiation, not selfrenewal, of human embryonic stem cells and is repressed by Oct4. Proc Natl Acad Sci U S A 2012;109:4485-90.

51. Paul I, Bhattacharya S, Chatterjee A, Ghosh MK. Current Understanding on EGFR and Wnt/beta-Catenin Signaling in Glioma and Their Possible Crosstalk. Genes Cancer 2013;4:427-46.

52. Zhao XD, Han X, Chew JL, Liu J, Chiu KP, Choo A, Orlov YL, Sung WK, Shahab A, Kuznetsov VA, Bourque G, Oh S, Ruan Y, et al. Whole-genome mapping of histone H3 Lys4 and 27 trimethylations reveals distinct genomic compartments in human embryonic stem cells. Cell Stem Cell 2007;1:286-98.

53. Jiang W, Wang J, Zhang Y. Histone H3K27me3 demethylases KDM6A and KDM6B modulate definitive endoderm differentiation from human ESCs by regulating WNT signaling pathway. Cell Res 2013;23:122-30.

54. Foroni C, Broggini M, Generali D, Damia G. Epithelialmesenchymal transition and breast cancer: role, molecular mechanisms and clinical impact. Cancer Treat Rev.2012;38:689-97.

55. William C. Reinhold, Mark A. Reimers, Philip Lorenzi, Jennifer Ho, Uma T. Shankavaram, Micah S. Ziegler, Kimberly J. Bussey, Satoshi Nishizuka, Ogechi Ikediobi, Yves G. Pommier, John N. Weinstein. Multifactorial regulation of E-Cadherin expression: an integrative study. Mol Cancer Ther.2010;9:1-16. 